\title{
Effect of osteopathic manipulative treatment on gastrointestinal function and length of stay of preterm infants: an exploratory study
}

\author{
Gianfranco Pizzolorusso ${ }^{1 *}$, Patrizia Turi ${ }^{1}$, Gina Barlafante ${ }^{2}$, Francesco Cerritelli ${ }^{1}$, Cinzia Renzetti ${ }^{2}$, \\ Vincenzo Cozzolino ${ }^{2}$, Marianna D'Orazio ${ }^{2}$, Paola Fusilli ${ }^{3}$, Fabrizio Carinci ${ }^{1}$ and Carmine D'Incecco $^{3}$
}

\begin{abstract}
Background: Organizational improvement of neonatal intensive care units requires strict monitoring of preterm infants, including routine assessment of physiological functions of the gastrointestinal system and optimized procedures for the definition of appropriate discharge timing.

Methods: We conducted a prospective study on the effect of osteopathic manipulative treatment in a cohort of $\mathrm{N}$ $=350$ consecutive premature infants admitted to a neonatal intensive care unit without any major complication between 2005 and 2008. In addition to ordinary care, N = 162 subjects received osteopathic treatment. Endpoints of the study were differences between study and control groups in terms of excessive length of stay and gastrointestinal symptoms, defined as the upper quartiles in the distribution of the overall population. Statistical analysis was based on crude and adjusted odds ratios from multivariate logistic regression.

Results: Baseline characteristics were evenly distributed across treated/control groups, except for the rate of infants unable to be oral fed at admission, significantly higher among those undergoing osteopathic care $(p=.03)$. Osteopathic treatment was significantly associated with a reduced risk of an average daily occurrence of gut symptoms per subject above $.44(\mathrm{OR}=0.45 ; 0.26-0.74)$. Gestational age lower or equal to 32 weeks, birth weight lower or equal to 1700 grams and no milk consumption at admission were associated with higher rates of length of stay in the unit of at least 28 days, while osteopathic treatment significantly reduced such risk $(O R=0.22 ; 0.09-0.51)$.

Conclusions: In a population of premature infants, osteopathic manipulative treatment showed to reduce a high occurrence of gastrointestinal symptoms and an excessive length of stay in the NICU. Randomized control studies are needed to generalize these results to a broad population of high risk newborns.
\end{abstract}

\section{Background}

Significant improvements in neonatal technology utilized in neonatal intensive care units (NICUs) over the last 2 decades, along with evidence-based care guidelines, have significantly improved hospitalization and survival for both low birth weight (LBW) infants and the residual preterm population, albeit at a high cost. A major proportion of pediatric hospital stays in the United States is attributable to neonatal conditions that rank among the most expensive items in the list of services provided for

\footnotetext{
* Correspondence: gianfranco.pizzolorusso@gmail.com

'EBOM - European Institute for Evidence Based Osteopathic Medicine, viale Unità d'Italia 1, 66100 Chieti, Italy

Full list of author information is available at the end of the article
}

children [1]. The average cost per infant is highest for preterm newborns with gestational age (GA) between 24-31 weeks, and next highest for those between 32-36 weeks, as opposed to the general population [2]. Costs per surviving infant generally decrease with increasing GA. In the United States, preterm/LBW infants account for half the hospitalization costs of all newborns and one quarter of overall pediatric costs [3].

Length of stay (LOS) in NICUs is strongly associated with GA and birth weight [4]. Infants delivered at the earliest GA have the longest hospital stays, partly because of the higher incidence of medical complications in very low birth weight (VLBW) infants.

\section{() Biomed Central}


However, compared to term infants, premature infants are unique in their need to attain not only medical stability but also physiologic maturity, including adequate temperature control, cessation of apnea and bradycardia, and adequate feeding behavior, before they are safely discharged to home $[5,6]$.

Patterns of hospitalization of preterm infants are also associated with the presence of clinical symptoms of abnormal gastrointestinal function. In particular, vomit and regurgitation were found to be associated with increased esophageal acid occurrence among NICU patients [7], as well as gastric residuals (GR) [8], which can be linked to feeding behaviors and definitely improved by targeted feeding strategies.

In VLBW infants, feeding tolerance algorithms are based on pre-prandial GR volume measurement. High pre-prandial volumes of GR are regarded as significant markers of feeding intolerance [9]. Previous studies in NICUs show that neonates under stress have a higher incidence of stress-induced gastric mucosal damage [10,11]. Functional constipation and hard stools are common conditions in both term and preterm infants, usually leading to changes in feeding formulas [12] and use of enemas in specific settings [13].

Non invasive treatments to improve feeding tolerance and to reduce clinical complications of premature infants may represent a convenient option in the absence of standard procedures for specific subgroups of patients.

The present report describes the activity of a research team investigating the effects of Osteopathic Manipulative Treatment (OMT) in preterm infants, including monitoring of physiological functions of the gastrointestinal system and LOS.

\section{Methods}

\section{Objective and endpoints of the study}

To evaluate the efficacy of OMT on premature infants during hospitalization. Endpoints of the study were differences between study and control groups in terms of changes in gastrointestinal function and LOS.

Primary endpoints were measured over the entire period of NICU hospitalization as follows:

I. High frequency of gut symptoms, defined as the upper quartile of the average number of episodes of vomit, regurgitation, GR and enema per measurement visit per subject.

II. Excess duration of LOS, defined as the upper quartile of LOS in NICU per subject.

\section{Study Design and Population}

The study was based on a non randomized, longitudinal observational design investigating outcomes in a cohort of newborns admitted to the NICU of the main public hospital in Pescara, Abruzzo, Italy.

Eligible subjects included all infants consecutively admitted between January 2005 to June 2008 ( $\mathrm{N}=663)$. A total of $\mathrm{N}=359$ passed the following exclusion criteria: GA less than 29 weeks, or greater than 37 weeks; osteopathic treatment performed more than 14 days after birth; newborn transferred to/from other hospital/unit; newborn from an HIV seropositive and/or drug addicted mother; newborn with any of the following clinical conditions: genetic disorders, congenital abnormalities, cardiovascular abnormalities, neurological disorders; proven or suspected necrotizing enterocolitis with or without gastrointestinal perforation; proven or suspected abdominal obstruction; pre- and/or post- surgery patients; pneumoperitoneum and/or atelectasis. Among the 304 subjects excluded, 232 infants had a GA below 29 or above 37, while 78 subjects presented with severe clinical conditions.

After enrollment, 4 additional infants were dropped because of an unrecorded birth weight, and 5 infants (2 from the study group; 3 from the control group) because of complications arising during hospitalization.

The final total number of infants analyzed in this study was 350 .

A total of 188 preterm infants were non-randomly assigned to routine neonatal care; while 162 subjects received routine care plus OMT. All patients from both groups were transferred from the delivery and/or operating room to the NICU immediately after birth.

No prior manipulation provided by any physical and/or massage therapist was performed on any infant.

\section{Data collection}

Data collection was performed by undergraduate osteopaths from the Accademia Italiana Osteopatia Tradizionale (AIOT). Measurements were recorded twice a week (Tuesdays and Fridays) based upon NICU's clinical charts completed by nursing staff who provided care on the same day.

Additional infant information was included: date of birth, admission/discharge from NICU, GA at birth (based on best obstetrical estimate), birth weight at admission and discharge, formula and/or breast milk intake volume. Gastrointestinal function was measured as regurgitation (defined as the passage of refluxed gastric contents into the oral pharynx), or vomiting (defined as the expulsion of the refluxed gastric contents from the mouth, i.e. feeding tolerance), or GR finding (milky, bilious and bloody; measured only on infants with oro/ naso-gastric tube, recorded as present/not present), frequency of stooling and enema administration per patient care encounter. A neurological/developmental evaluation at entry/discharge was not available for this study as it 
does not constitute part of routine assessment in the NICU.

Data were directly entered on an Excel spreadsheet

\section{Osteopathic Manipulative Treatment}

Osteopathic treatment was administered to the intervention group on Tuesdays and Fridays. Subjects in the study arm received osteopathic care within 14 days after birth, regardless of the application of any other procedure (i.e. mechanical ventilation, blood transfusion or phototherapy).

OMT was performed by a group of osteopaths certified by the Registro degli Osteopati d'Italia with at least five years of clinical experience.

Treatment duration ranged between 20-30 minutes. The infant's entire body was evaluated and manipulative procedures were provided as indicated by the osteopathic palpatory structural examination results. Osteopaths performing OMT were trained to use only indirect and fluidic techniques which included: indirect myofascial, sutural spread, balanced membranous tension and balanced ligamentous tension (according to teachings of William Garner Sutherland, DO, and others [14]).

\section{Clinical procedures and discharge strategy}

Feeding regimen, feeding strategies and enema administration were based on the application of standard international guidelines to both study arms $[13,15]$. As distinct from UK/US hospitals, enema prescription used by the study NICU included five percent glucose glycerin enemas (10:1 mixture, $5 \mathrm{~mL} / \mathrm{kg}$ ), administered twice a day, until infants spontaneously expel at least 1 stool per day.

Physiological conditions required for discharge included: maintenance of body heat at room temperature, coordinated sucking, swallowing, and breathing while feeding; sustained pattern of weight gain; and stability of cardiorespiratory function (no episodes of apnea/bradycardia for 2-5 days, free of supplemental oxygen support) [6].

\section{Statistical analysis}

Main results are expressed in terms of odds ratios between each level of a potential risk factor and a set reference category (R.C.), with primary endpoints classified as binary outcomes (low/high).

Potential confounders included the following characteristics (categories): gender, GA $(\leq 32 ;>32-\leq 35 ;>35$ weeks), birth weight ( $\leq 1700 ;>1700-\leq 2200 ;>2200$ grams), oral feeding at admission ( $\mathrm{No} / \mathrm{Yes}$ ).

Univariate statistical tests included formal tests of the differences between study and control groups using chisquare for categorical variables and unpaired t-tests for continuous measurements.

Multivariate logistic regression was used to estimate the independent effect of OMT on primary outcomes, simultaneously adjusting for all potential confounders. Statistical significance was based on a probability level $(\alpha)$ equal to 0.05 . Results were expressed in terms of point estimates (odds ratios: OR) and 95\% confidence intervals (C.I.). All analyses were performed using the statistical programming language $R$ [16].

\section{Results}

Univariate statistical analyses are shown in Table 1. No significant imbalances were found among treated and control groups in terms of main characteristics measured at admission, except for milk at admission ( $\mathrm{p}=$ 0.03 ), showing a higher percentage of infants unable to be oral fed at entry into this study among those treated with OMT.

Upper quartiles led to the definition of the following thresholds for the outcomes of interest:

1) average daily occurrence of gut symptoms per subject above .44;

2) LOS of at least 28 days.

Results for gastrointestinal function are shown in Table 2. None of the risk factors considered as potential correlates were found to be associated with an high rate of gut symptoms, except for OMT $(\mathrm{OR}=0.45 ; 0.27$ 0.74). Multivariate logistic regression confirmed OMT to be independently associated with a $55 \%$ reduction of gastrointestinal symptoms (Adjusted OR $=0.45 ; 0.26$ $0.74)$.

Results for LOS are reported in Table 3. Univariate odds ratios showed the following categories to be associated with increased rates of LOS equal or above 28

\section{Table 1 General characteristics of the study population}

\begin{tabular}{|c|c|c|c|}
\hline & Study Group & Control Group & $p$ value \\
\hline $\mathrm{N}$ & $162(46.3)$ & $188(53.7)$ & \\
\hline \multicolumn{4}{|l|}{ Gender } \\
\hline Females & $81(50.0)$ & $89(47.3)$ & \\
\hline Males & $81(50.0)$ & $99(52.7)$ & 0.70 \\
\hline \multicolumn{4}{|l|}{ Gestational Age } \\
\hline$\leq 32$ & $39(24.1)$ & $43(22.9)$ & \\
\hline$>32, \leq 35$ & $69(42.6)$ & $72(38.3)$ & \\
\hline$>35$ & $54(33.3)$ & $73(38.8)$ & 0.56 \\
\hline \multicolumn{4}{|l|}{ Weight (grams) } \\
\hline \multicolumn{4}{|l|}{ At Birth } \\
\hline$\leq 1700$ & $27(16.7)$ & $36(19.2)$ & \\
\hline$>1700, \leq 2200$ & $62(38.3)$ & $63(33.5)$ & \\
\hline$>2200$ & $73(45.0)$ & 89 (47.3) & 0.62 \\
\hline At Admission* & $2148(486.7)$ & $2212(562.3)$ & 0.25 \\
\hline \multicolumn{4}{|c|}{ Oral feeding at admission } \\
\hline No & $129(79.6)$ & $129(68.6)$ & \\
\hline Yes & $33(20.4)$ & $59(31.4)$ & 0.03 \\
\hline
\end{tabular}

Numbers in Table are $\mathrm{N}(\%), \mathrm{p}$ values from Chi Square test

* = mean, (standard deviation); $p$ value from $t$ test 
Table 2 Results for Average Daily Gut Symptoms: Crude and Adjusted Odds Ratios from Multivariate Logistic Regression

\begin{tabular}{|c|c|c|c|c|c|c|}
\hline & \multicolumn{2}{|c|}{ Average Daily Gut Symptoms* } & \multicolumn{2}{|c|}{ Univariate O.R. } & \multicolumn{2}{|c|}{ Adjusted O.R } \\
\hline & $\leq 0.44$ & $>0.44$ & O.R. $(95 \% \mathrm{Cl})$ & $p>\mid \chi^{2}$ & O.R. $(95 \% \mathrm{Cl})$ & $p>\mid \chi^{2}$ \\
\hline N & $262(74.9)$ & $88(25.1)$ & & & & \\
\hline \multicolumn{7}{|l|}{ Gender } \\
\hline Females [R.C] & $129(75.9)$ & $41(24.1)$ & 1 & - & 1 & - \\
\hline Males & $133(73.9)$ & $47(26.1)$ & $1.11(0.68-1.80)$ & 0.759 & $1.08(0.65-1.79)$ & 0.777 \\
\hline \multicolumn{7}{|l|}{ Gestational Age } \\
\hline$\leq 32$ & $57(69.5)$ & $25(30.5)$ & $1.20(0.65-2.21)$ & 0.670 & $1.02(0.43-2.40)$ & 0.965 \\
\hline$>32, \leq 35$ & $112(79.4)$ & $29(20.6)$ & $0.71(0.40-1.25)$ & 0.293 & $0.72(0.39-1.32)$ & 0.292 \\
\hline$>35[$ R.C $]$ & $93(73.2)$ & $34(26.8)$ & 1 & - & 1 & - \\
\hline \multicolumn{7}{|l|}{ Birth Weight (grams) } \\
\hline$\leq 1700$ & $39(67.2)$ & 19 (32.8) & $1.54(0.80-2.96)$ & 0.265 & $1.39(0.55-3.46)$ & 0.481 \\
\hline$>1700, \leq 2200$ & $100(76.9)$ & $30(23.1)$ & $0.95(0.55-1.63)$ & 0.952 & $1.03(0.55-1.93)$ & 0.927 \\
\hline$>2200[$ R.C $]$ & $123(75.9)$ & $39(24.1)$ & 1 & - & 1 & - \\
\hline \multicolumn{7}{|c|}{ Oral feeding at admission } \\
\hline No & $192(74.4)$ & $66(25.6)$ & $1.09(0.63-1.90)$ & 0.860 & $1.18(0.67-2.13)$ & 0.583 \\
\hline Yes [R.C] & $70(76.1)$ & $22(23.9)$ & 1 & - & 1 & - \\
\hline \multicolumn{7}{|l|}{ OMT } \\
\hline No $[R . C]$ & $128(68.1)$ & $60(31.9)$ & 1 & - & 1 & - \\
\hline Yes & $134(82.7)$ & $28(17.3)$ & $0.45(0.27-0.74)$ & 0.002 & $0.45(0.26-0.74)$ & 0.002 \\
\hline
\end{tabular}

R.C. = Reference Category

* No. of episodes of Vomit, Regurgitation, Gastric residual and Enema

days: $\mathrm{GA} \leq 32$ weeks $(\mathrm{OR}=38.10 ; 16.40-88.20$; R.C.:GA $>35$ weeks), birth weight $\leq 1700$ gm vs $>2200$ gm (OR

$=120.60 ; 42.70-340.60)$ and birth weight $>1700 \mathrm{gm}, \leq$ 2200 gm $(\mathrm{OR}=5.80 ; 2.40-13.80$; R.C.: birth weight $>$
$2200 \mathrm{gm}$ ), oral feeding at admission (OR $=2.85 ; 1.44$ 5.66) and OMT $(\mathrm{OR}=0.51 ; 0.30-0.85)$. Multivariate logistic regression showed similar patterns, confirming an independent effect of OMT, simultaneously adjusted

Table 3 Results for Length of Stay (LOS): Crude Odds Ratios (p value from Cochran Mantel Haenszel Chi Square Test of Zero Correlation) and Adjusted Odds Ratios from Multivariate Logistic Regression (p value from partial test on regression coefficient)

\begin{tabular}{|c|c|c|c|c|c|c|}
\hline & \multicolumn{2}{|c|}{ LOS (days) } & \multicolumn{2}{|c|}{ Univariate O.R. } & \multicolumn{2}{|c|}{ Adjusted O.R. } \\
\hline & $<28$ & $\geq 28$ & O.R. $(95 \% \mathrm{Cl})$ & $p>\mid \chi^{2}$ & O.R. $(95 \% \mathrm{Cl})$ & $p>\mid \chi^{2}$ \\
\hline N & $267(76.3)$ & $83(23.7)$ & & & & \\
\hline \multicolumn{7}{|l|}{ Gender } \\
\hline Females [R.C] & $128(75.3)$ & $42(24.7)$ & 1 & - & 1 & - \\
\hline Males & $139(77.2)$ & $41(22.8)$ & $0.90(0.55-1.47)$ & 0.765 & $1.40(0.63-3.10)$ & 0.412 \\
\hline \multicolumn{7}{|l|}{ Gestational Age } \\
\hline$\leq 32$ & $21(25.6)$ & $61(74.4)$ & $38.10(16.40-88.20)$ & $<0.001$ & $10.90(3.53-33.72)$ & $<0.001$ \\
\hline$>32, \leq 35$ & $128(90.8)$ & $13(9.2)$ & $1.33(0.55-3.22)$ & 0.680 & $0.76(0.27-2.15)$ & 0.609 \\
\hline$>35[$ R.C $]$ & $118(92.9)$ & $9(7.1)$ & 1 & - & & \\
\hline \multicolumn{7}{|l|}{ Birth Weight (grams) } \\
\hline$\leq 1700$ & $9(15.5)$ & $49(84.5)$ & $120.60(42.70-340.60)$ & $<0.001$ & $43.23(11.63-160.66)$ & $<0.001$ \\
\hline$>1700, \leq 2200$ & $103(79.2)$ & $27(20.8)$ & $5.80(2.40-13.80)$ & $<0.001$ & $3.01(1.05-8.68)$ & 0.041 \\
\hline$>2200[$ R.C $]$ & $155(95.7)$ & $7(4.3)$ & 1 & - & 1 & - \\
\hline \multicolumn{7}{|c|}{ Oral feeding at admission } \\
\hline No & $186(72.1)$ & $72(27.9)$ & $2.85(1.44-5.66)$ & 0.003 & $3.11(1.05-9.25)$ & 0.041 \\
\hline Yes [R.C] & $81(88.0)$ & $11(12.0)$ & 1 & - & 1 & - \\
\hline \multicolumn{7}{|l|}{ OMT } \\
\hline No $[R . C]$ & $133(70.7)$ & $55(29.3)$ & 1 & - & 1 & - \\
\hline Yes & $134(82.7)$ & $28(17.3)$ & $0.51(0.30-0.85)$ & 0.012 & $0.22(0.09-0.51)$ & $<0.001$ \\
\hline
\end{tabular}

R.C. = Reference Category 
for all factors, corresponding to more than a $75 \%$ reduction in excessive LOS (Adjusted OR $=0.22 ; 0.09-0.51$ )

\section{Discussion}

The main objective of this exploratory study was to investigate the effects of OMT in a population of premature infants in terms of gastrointestinal functions and LOS.

The medical literature lacks information of any potential benefits of complementary treatments in this area. To the best of the authors' knowledge, OMT in premature newborns has never been documented by pediatric specialty journals. Studies carried out in pediatric patients suggested positive effects of OMT in very young children [17-19]. In the broader field of manual therapy, specialists of massage therapy and kinesthetic stimulation showed positive results in premature infants [20]. However, such findings were inconsistent and obtained with heterogeneous methods, showing only minimal differences in terms of clinical significance [20,21].

The present study suggests that OMT may reduce the occurrence of frequent symptoms of abnormal gastrointestinal functionality.

Precise mechanisms for such positive effects generated by OMT are difficult to specify, but several hypotheses can be offered on the basis of neurological, tissue and neuroendocrine factors.

In terms of neurology, there is evidence of an association between autonomic nervous system function and OMT, showing a significant direct relation between myofascial release technique and modifications in the autonomic nervous system activity [22].

Regarding the interaction between OMT and tissue modification, in-vitro models highlight a possible decrease in the production of inflammatory factors [23].

A possible role of neuroendocrine factors can be hypothesized as indicated by the evidence of the effect of OMT on pain biomarker modification in patients affected by low back pain [24].

This study also shows that a significantly higher rate of premature infants receiving osteopathic care can be discharged before 28 days regardless of gender, GA, birth weight and oral feeding at admission.

Such a result may have important implications for the optimization of health care in premature infants. Focusing on the percentage of patients discharged before a given threshold, rather than looking at the average reduction in LOS, may be very relevant for health optimization and cost control. Reducing the rate of long stays would reduce the number of patients in the NICU, allowing for more cribs to become simultaneously available for those infants who require specialized care.

From an epidemiological point of view, the potential benefit may also spread beyond discharge, considering that hospitalization can influence nutrition [25] and morbidity of gastrointestinal infections [26].

An understanding of the differential advantage of OMT on specific subgroups, in particular within specified classes of GA, will require ad hoc studies with an adequate sample size. In the present study, it was not possible to perform subgroups analyses on subjects with very low GA, due to the very limited number of patients available for enrollment.

Finally, some intrinsic limitations of the present study need to be outlined.

This report is based on measurements implemented at the local NICU at the start of the study. Additional relevant confounding variables such as maternal/delivery factors (including breast feeding), respiratory support, method of feeding and gastric emptying time could not be included in this study.

Treatment allocation was neither randomized nor structured, as it was based on matters of convenience within the constraints of the proposed two days per week of osteopathic care. Furthermore, due to the current logistics and procedures it was not possible to "blind" nurses and neonatologists to treatment regimen.

This study, which was conducted in only one NICU, cannot capture the intrinsic variability of organizational strategies across multiple clinical centers managing the complexities of the overall population of newborn infants.

From a methodological point of view, sample size was not based on formal power estimation, treatment was not allocated using a random procedure, and the population of preterm infants may not be representative of the entire population of cases.

The above limitations affect our ability to check for bias and duly rely on the precision of our estimates. In other terms, both the size of the effect of OMT (point estimate) and its level of uncertainty (95\% confidence interval) are more likely to be inconsistent with further results obtainable under more general conditions.

To evaluate the efficacy of OMT more studies are required using formal experimental methods, such as randomized and placebo controlled clinical trials. The best endpoint of a well designed three armed study would be the difference between the sham and the actual treatment. However, to make it possible, osteopaths should collaborate with NICU managers to revise the application of operational procedures, so that OMT can be smoothly applied on large populations, across multiple clinical sites.

Despite the above limitations, and given the current lack of information on the possible effects of OMT in preterm infants, the finding of this report sets an interesting ground for new developments. Among these, the standard measurement of all relevant parameters represents an essential aspect that deserves attention for future 
investigations. Key characteristics and outcomes that can be easily monitored on a daily basis by clinicians, nurses and even parents of preterm infants have been identified. Their adoption for the construction of electronic data base registers can offer a sustainable means to improve both analysis and management of NICU activity, allowing to carry out more detailed exploratory studies while providing a basis for ongoing trials.

\section{Conclusion}

The study suggests that osteopathic treatment may reduce a high occurrence of gastrointestinal symptoms and the rates of long-term stays.

Randomized control studies are needed to confirm these results and to generalize them to a broader population of high risk newborns.

\author{
Abbreviations \\ NICU: neonatal intensive care unit; VLBW: very low birth weight; LBW: low \\ birth weigh; GA: gestational age; LOS: length of stay; GER: gastroesophageal \\ reflux; GR: gastric residual; OMT: osteopathic manipulative treatment; OR: \\ odd ratio.
}

\section{Author details}

${ }^{1}$ EBOM - European Institute for Evidence Based Osteopathic Medicine, viale Unità d'Italia 1, 66100 Chieti, Italy. ${ }^{2}$ AIOT - Accademia Italiana Osteopatia Tradizionale, via Prati 29, 65124 Pescara, Italy. ${ }^{3}$ Unità di Terapia Intensiva Neonatale - Ospedale Civile Spirito Santo, Via Renato Paolini 45, 65124 Pescara, Italy.

\section{Authors' contributions}

$G B$ and $C D$ conceived the idea of the study. GP, GB, VC, CR and FCE participated in the design of the study and its coordination. GP, PT, MD, FCE and PF coordinated and performed the data collection. GP and FCA drafted the manuscript. FCA performed the statistical analysis. All authors read and approved the final manuscript.

\section{Competing interests}

The authors declare that they have no competing interests.

Received: 16 November 2010 Accepted: 28 June 2011

Published: 28 June 2011

\section{References}

1. Owens PL, Thompson J, Elixhauser A, Ryan K: Care of Children and Adolescents in U.S. Hospitals.Edited by: Rockville, MD. Agency for Healthcare Research and Quality; 2003:, Report HCUP Fact Book No. 4; AHRQ Publication 04-0004.

2. Clements KM, Barfield WD, Ayadi MF, Wilber N: Preterm birth-associated cost of early intervention services: an analysis by gestational age. Pediatrics 2007, 119(4):e866-74, Epub 2007 Mar 5.

3. Russell RB, Green NS, Steiner CA, Meikle S, Howse JL, Poschman K, Dias T, Potetz L, Davidoff MJ, Damus K, Petrini JR: Cost of hospitalization for preterm and low birth weight infants in the United States. Pediatrics 2007, 120(1):e1-9.

4. Bakewell-Sachs S, Medoff-Cooper B, Escobar GJ, Silber JH, Lorch SA: Infant functional status: the timing of physiologic maturation of premature infants. Pediatrics 2009, 123(5):e878-86.

5. Eichenwald EC, Blackwell M, Lloyd JS, Tran T, Wilker RE, Richardson DK: Inter-neonatal intensive care unit variation in discharge timing: influence of apnea and feeding management. Pediatrics 2001, 108(4):928-33.

6. American Academy of Pediatrics: Hospital discharge of the high-risk neonate-proposed guidelines. Pediatrics 1998, 102:411-7.

7. Mezzacappa MA, Rosa AC: Clinical predictors of abnormal esophageal pH monitoring in preterm infants. Arq Gastroenterol 2008, 45(3):234-8.
8. Mihatsch WA, von Schoenaich P, Fahnenstich $H$, Dehne N, Ebbecke $H$ Plath C, von Stockhausen HB, Muche R, Franz A, Pohlandt F: The significance of gastric residuals in the early enteral feeding advancement of extremely low birth weight infants. Pediatrics 2002, 109(3):457-9.

9. Bertino E, Giuliani F, Prandi G, Coscia A, Martano C, Fabris C: Necrotizing enterocolitis: risk factor analysis and role of gastric residuals in very low birth weight infants. J Pediatr Gastroenterol Nutr 2009, 48(4):437-42.

10. Mäki M, Ruuska T, Kuusela AL, Karikoski-Leo R, Ikonen RS: High prevalence of asymptomatic esophageal and gastric lesions in preterm infants in intensive care. Crit Care Med 1993, 21(12):1863-7.

11. Kuusela AL, Mäki M, Ruuska T, Laippala P: Stress-induced gastric findings in critically ill newborn infants: frequency and risk factors. Intensive Care Med 2000, 26(10):1501-6.

12. Duman N, Utkutan S, Ozkan H, Ozdoğan S: Are the stool characteristics of preterm infants affected by infant formulas? Turk J Pediatr 2000, 42(2):138-44.

13. Mihatsch WA, Franz AR, Högel J, Pohlandt F: Hydrolyzed protein accelerates feeding advancement in very low birth weight infants. Pediatrics 2002, 110(6):1199-203.

14. Magoun HI: Osteopathy in the Cranial Field.Edited by: Kirksville, Mo. Journal Printing Co;, 31976.

15. Nutrition Toolkit: 2008 [http://www.cpqcc.org/documents/341/download], Accessed June 24th 2010.

16. The R Development Core Team, R: A Language and Environment for Statistical Computing. [http://cran.r-project.org/doc/manuals/refman.pdf], Accessed June 24th 2010.

17. Hayden C, Mullinger B: A preliminary assessment of the impact of cranial osteopathy for the relief of infantile colic. Complement Ther Clin Pract 2006, 12(2):83-90.

18. Vandenplas $Y$, Denayer $E$, Vandenbossche $T$, Vermet $L$, Hauser $B$, Deschepper J, Engelen A: Osteopathy may decrease obstructive apnea in infants: a pilot study. Osteopath Med Prim Care 2008, 19(2):8.

19. Philippi $H$, Faldum A, Schleupen A, Pabst B, Jung T, Bergmann H, Bieber I, Kaemmerer C, Dijs P, Reitter B: Infantile postural asymmetry and osteopathic treatment: a randomized therapeutic trial. Dev Med Child Neurol 2006, 48(1):5-9.

20. Massaro AN, Hammad TA, Jazzo B, Aly H: Massage with kinesthetic stimulation improves weight gain in preterm infants. J Perinatol 2009, 29(5):352-7.

21. Vickers A, Ohlsson A, Lacy JB, Horsley A: Massage for promoting growth and development of preterm and/or low birth-weight infants. Cochrane Database Syst Rev 2004, 2: CD000390, Review.

22. Henley CE, Ivins D, Mills M, Wen FK, Benjamin BA: Osteopathic manipulative treatment and its relationship to autonomic nervous system activity as demonstrated by heart rate variability: a repeated measures study. Osteopath Med Prim Care 2008, 5(2):7.

23. Meltzer KR, Standley PR: Modeled repetitive motion strain and indirect osteopathic manipulative techniques in regulation of human fibroblast proliferation and interleukin secretion. J Am Osteopath Assoc 2007, 107(12):527-36.

24. Degenhardt BF, Darmani NA, Johnson JC, Towns LC, Rhodes DC, Trinh C, McClanahan B, DiMarzo V: Role of osteopathic manipulative treatment in altering pain biomarkers: a pilot study. J Am Osteopath Assoc 2007, 107(9):387-400.

25. Kirchner L, Jeitler $V$, Waldhör T, Pollak A, Wald M: Long hospitalization is the most important risk factor for early weaning from breast milk in premature babies. Acta Paediatr 2009, 98:981-984.

26. Kramer MS, Kakuma R: Optimal duration of exclusive breastfeeding. Cochrane Database Syst Rev 2002, , 1: CD003517.

doi:10.1186/2045-709X-19-15

Cite this article as: Pizzolorusso et al:: Effect of osteopathic manipulative treatment on gastrointestinal function and length of stay of preterm infants: an exploratory study. Chiropractic \& Manual Therapies 2011 19:15. 